\title{
The impact of COVID-19 threats, and preventive chain of action: molecule to medicine
}

\author{
Mohammed H. Alqahtani, Diane E. Heck and Hong Duck Kim* \\ Department of Public Health, School of Health Sciences and Practice, New York Medical College, Valhalla NY.
}

GSC Biological and Pharmaceutical Sciences, 2021, 17(03), 119-123

Publication history: Received on 06 August 2021; revised on 13 December 2021; accepted on 15 December 2021

Article DOI: https://doi.org/10.30574/gscbps.2021.17.3.0232

\begin{abstract}
The emergence of the novel coronavirus (SARS-CoV-2) had affected us significantly from the individual level, to nationwide and global with a big loss of finances, and the freezing of various factories, schools, and transportation in communities The pandemic started with anxiety and a loss of health guidance and policies due to the unknown causes of viral transmission to human features as well as a high infection rate with low mortality It remains the original source of Covid-19 where it comes from and what is the reality of real viral entities and its origin such as natural born and recombinant viral variants in the case of COVID-19 pandemic. This sentence is unclear. In this short perspective article, we address some issues of risk assessment and management issues using molecular-based decision tools which may benefit or provide future drills to counteract health and clinic safety against a viral pandemic. Every pandemic gives us life threatening lessons on previous and disconnected human networks due to uncertainty of viral infection, which we learned from this COVID-19 pandemic case as well. It gives us some insight on how to rebuild our community regarding the strength of public health and the integration of science tools into the early phase of medical application, such as the role of molecular diagnostics through educational engagement. To promote the value of awareness with solid knowledge-based communication and to develop resilient preventive solutions for supply chains or prevention, the systematic practice of connectivity through visual format using multidimensional data outcomes could help reconsider the leverage of molecules as a bridge for the improvement and application of updated scientific tools of prediction precisely to identify unknown pathogens encompass rigor community-based activity likelihood sensitivity and resistance to pathogen infiltrated society in the future.
\end{abstract}

Keywords: SARS-CoV-2; COVID-19; mRNA vaccine; Detection of virus antigen; Neutralization antibody; Surveillance system; Molecular-based risk assessment skills; Omics

\section{Introduction}

For centuries, humans have been affected by severe epidemics associated with infectious diseases. The implications of these epidemics have significantly affected public health and claimed multiple lives. For instance, the bubonic plague, or the Black Death, claimed approximately 50 percent of the European population during the Middle Ages [8]. In the Americas, smallpox also adversely affected the Native American population, promoting European conquest. However, an epidemic may exacerbate and gravitate across particular geographical regions to have a global influence. In cases where the epidemic spreads worldwide, it is designated a pandemic. The 1918 influenza and the acquired immunodeficiency syndrome (AIDS) are examples of pandemics that severely affected public health. The 1918 Influenza pandemic occurred during World War I when populations were displaced and lacked the resources to address a medical emergency [8]. The absence of a medical solution, such as antibiotics for treatment and inadequate healthcare solutions, also caused millions of deaths associated with 1918 Influenza. Therefore, the mitigation and management of pandemics are not new in the public health domain.

\footnotetext{
${ }^{*}$ Corresponding author: Hong Duck Kim

Department of Public Health, School of Health Sciences and Practice, New York Medical College, Valhalla NY. 


\section{Epidemiology and Diagnosis}

The emergence of a new pandemic in 2019, the novel coronavirus (SARS-CoV-2), has also presented a public health challenge. The first case of coronavirus disease (SARS-CoV-2) was reported in Wuhan city, Hubei Province, in the People's Republic of China [11]. The disease has since spread to other countries, with the World Health Organization (2021) reporting its existence in over 200 countries worldwide [16, 17]. The novel coronavirus (SARS-CoV-2) is an enveloped, positive single-stranded RNA virus that infects humans [1]. Velevan and Meyer (2020) highlight that the first documentation of coronaviruses was done in 1966 by Tyrell and Bynoe in a study to cultivate viruses from patients with particular cold cases of flu [15]. The virus acquired its name based on its morphology, which presents itself as a spherical virion with a shell and surface projections similar to a solar corona. Coronaviruses are subdivided into four sub-families: alpha-coronavirus, beta-coronavirus, gamma-coronavirus, and delta-coronavirus. Unlike alpha-coronaviruses that originate from bats, the other sub-families (beta-coronaviruses, gamma-corona-viruses, and delta-coronaviruses) are derived from birds and pigs. The genome of the coronavirus may vary, ranging between $26 \mathrm{~kb}$ and $32 \mathrm{~kb}$. While the alpha-coronaviruses are asymptomatic and may fail to exhibit any significantly mild effects, the beta-coronaviruses are fatal since they result in severe infections. The novel coronavirus (SARS-CoV-2) portrays a close relationship with the (SARS-CoV) virus, and is therefore categorized under lineage B of the beta-coronaviruses. The gene structure of the SARS-CoV virus is comprised of a nucleocapsid protein $(\mathrm{N})$, a membrane protein, a spike protein (S), and a membrane glycoprotein (M) [18]. It also encodes another glycoprotein membrane (HE) that occurs in the HCoV-OC43 and HKU1 beta-coronaviruses.

According to Majumder and Mandl (2020), the novel coronavirus's incubation period (SARS-CoV-2) is 5.2 days [12]. The first clinical sign that was identified in novel coronavirus (SARS-CoV-2) patients is pneumonia. Since the novel coronavirus infection (SARS-CoV-2) is acute, various symptoms may manifest in fever, persistent coughs, and fatigue. Kirkcaldy et al. (2020) also posits that gastrointestinal symptoms have been observed among young children [10]. The novel coronavirus (SARS-CoV-2) impairs the respiratory system by causing shortness of breath, sore throat, hemoptysis, dry cough, and chest pains [12]. In gastrointestinal cases, the symptoms may range from nausea, diarrhea to vomiting. Symptomatic patients begin to exhibit clinical manifestation of the novel coronavirus (SARS-CoV-2) after approximately a week. These symptoms include headaches, nasal congestion, fatigue, and fever. The progression of the nasal coronavirus may result in dyspnea where the patient has chest pain complaints. During observation through chest Xrays, novel coronavirus (SARS-CoV-2) patients exhibit patchy consolidation in the alveoli [18]. Also, lymphopenia is common in novel coronavirus patients (SARS-CoV-2).

\section{Lifestyle and Preventive Action}

According to the World Health Organization (2021), the novel coronavirus (SARS-CoV-2) has infected over 126 million people globally and resulted in more than 2 million deaths [16]. However, more than 100 million people have recovered from the disease through healthcare management. The effect of the novel coronavirus (SARS-CoV-2) has been felt worldwide, with lockdowns being initiated in many countries, including the United States of America [2]. This has significantly affected the health, lifestyle, and overall wellbeing of many individuals. Many healthcare professionals have advised that the suitable way of managing the virus is a collective effort to reduce infection by flattening the curve.

Since SARS-CoV-2 is communicable, individuals were forced to adopt a new lifestyle. The Centers for Disease Control and Prevention (2020) recommends wearing masks as an effective way of curbing the spread of COVID-19 [5,6]. The coronavirus, SARS-CoV-2, spreads through aerosols when respiratory droplets are ejected from an individual's body through coughing and sneezing [1]. The Centers for Disease Control and Prevention (2020) highlights that in addition to wearing masks, people should observe social distancing, and they should wash their hands with soap and water [6]. In instances where water is insufficient or unavailable, hand sanitizers may be utilized as an alternative. Social distancing refers to the provision of physical distance between individuals to prevent the spread of COVID-19. Individuals must stay at least six feet apart and avoid public gatherings to curb the spread of COVID-19. The essence of social distancing is to prevent inhalation of droplets from infected people as they sneeze or cough [11]. The SARS-CoV2 may stay alive on certain surfaces for hours depending on the sunlight and humidity, hence the significance of using soap and washing hands regularly.

Currently, there is no accredited treatment for COVID-19. The disease is managed through symptomatic strategies and supportive care [10]. These include ventilation, oxygen provision, maintaining blood pressure, and treating associated secondary illnesses. Due to the absence of a validated treatment option, individuals should protect themselves from SARS-CoV-2 by taking various preventive measures. For instance, constant monitoring of the body temperature and self-surveillance is crucial, especially for those with a history of travel from high infectivity areas. In case of infection, 
the World Health Organization (2020) guidelines dictate self-isolation and quarantine measures for fourteen days [16]. Healthcare professionals must also protect themselves from the virus while attending to novel coronavirus patients. They are required to equip themselves with personal protective equipment while attending to both probable and confirmed cases of COVID-19.

\section{Immunization}

The World Health Organization (2021) highlights the importance of immunization in the alleviation of infectious diseases $[16,17]$. The international agency explains that vaccines save millions of human lives annually by training the human body to target and fight off viruses and bacteria. After vaccination, the body develops a natural defense to destroy disease-causing pathogens, hence preventing infections. According to the World Health Organization report, as of $18^{\text {th }}$ February 2021, there had been a roll-out of at least seven different vaccines deployed across different countries worldwide (World Health Organization, 2021). According to Chu et al. (2020), frontline workers and vulnerable populations are given priority during vaccination [7].

In the United States of America, the Centers for Disease Control and Prevention (2020) recommended Moderna, PfizerBioNTech, and Johnson \& Johnson/Janssen as suitable vaccines for the population [6]. The Centers for Disease Control and Prevention (2021) posits that all COVID-19 vaccines approved in the U.S. are safe and effective [5]. It emphasizes that vaccines are vital in preventing the novel coronavirus (COVID-19) and reduces the risk of complications associated with the disease. For instance, the mRNA-1273 (Moderna) vaccine is comprised of a lipid nanoparticle-encapsulated mRNA-based vaccine that encapsulates a stabilized spike protein of acute SARS-CoV-2. The BioNTech/Pfizer (i.e., BNT162b2) and Moderna vaccines show high efficacy rates ranging between 94 percent and 95 percent in preventing symptomatic infection of the novel coronavirus (SARS-CoV-2) [13]. During the deployment of vaccination, safety monitoring systems are crucial to determine the extent of immunization and its significance in the target population.

\section{COVID-19 Vaccine Promotion}

The roll-out of the COVID-19 vaccine is dependent on the public response and their acceptance. According to a Pew Research Survey (2020), only 51 percent of all Americans indicated their willingness to receive the novel coronavirus (SARS-CoV-2) vaccine. The survey reported low acceptance among African Americans (32\%) and those with lower educational levels (47\% among high school graduates and less education) [9]. Oronsky et al. (2021) postulate that an effective COVID-19 vaccine program should be built on integrity, urgency, and confidence [14]. They highlight various strategies vital in achieving widespread vaccine uptake hence an effective COVID-19 vaccination program.

First, the COVID-19 vaccine should be free and accessible across multiple populations. Ideally, the exclusion of frictional factors such as sign-up programs and waiting time increases the services' uptake. The elimination of small copayments and service charges increases the healthcare programs' impact by reducing barriers such as time and economic hurdles [3]. Another strategy is the conditional provision of access to specific settings conditional to vaccination. For instance, most retail outlets in the United States have launched campaigns to provide services to customers with face masks and while observing social distancing. This may be replicated in the vaccination program to promote the uptake of the COVID-19 vaccine. Similarly, access to sensitive settings such as clinical healthcare facilities and congregate shelter facilities (including nursing homes) should be conditional on the COVID-19 vaccines. Also, certain settings that may be hotbeds of infections, such as schools and public facilities, should be provisional only for vaccinated individuals. The transformation of individual vaccination consent into public acts through legislative processes will also increase the uptake of the COVID-19 vaccine. The utilization of public endorsements through trusted leaders and celebrities may positively impact the novel coronavirus (SARS-CoV-2) vaccine among populations [3]. It will establish trust and build confidence among the public by alleviating anxiety and reducing tension.

\section{Conclusion}

In conclusion, perspectives issues: this life threating lesson from the COVID-19 pandemic would be valuable to improve the surveillance connectivity chain from prediction and prevention, and in rebuilding the emergence drills of the novel coronavirus (SARS-CoV-2) and several harmful viral threats which have previously impacted public healthcare in multiple ways of similar viruses. The examination of its implications on the public healthcare system is vital in comprehending the severity of the virus and establishing better support systems for the future. Nonetheless, preventive action and mitigation strategies are crucial in managing any pandemic [4]. These engagements directing preventive action and mitigation strategies go through precision method, for example molecular based multi-dimensional approach (i.e., risk management with improved measurement skills from genomics to protein level) which could benefit to define 
viral pathogens effectively and efficiently using genomic-based decision tools (i.e., real time PCR- or digital PCR- based detection, genotyping, profile of polymorphism, and whole genome or exome-derived NGS sequence data outcomes). To reduce the pandemic's burden using an alternative therapeutic approach (i.e., chemical inhibitors, and biological countermeasures such as monoclonal and/or polyclonal antibody therapy to neutralize the virus growth and physiological outcomes like COVID-19 derived symptoms) on healthcare systems, health workers performing their designated roles adequately by enabling molecular-oriented surveillance, and monitoring of molecular behavioral such as identification variants for preventing infiltration pattern from the individual to the community or global. The priority for healthcare providers and associated stakeholders is to ensure public safety by establishing early prediction and prevention for protecting human lives by developing a suitable health action with transparency, right direction, and secure potential countermeasures under an integrated and systematic network from detection to treatment to curb the coronavirus's spread (SARS-CoV-2).

\section{Compliance with ethical standards}

\section{Acknowledgments}

We thank Peter Astriab for his invaluable help checking with grammatical errors, typos, and his tireless suggestion to improve the contents.

\section{Disclosure of conflict of interest}

No conflict financial authors declared.

\section{References}

[1] Abdulla Hama Salih M. An overview on the pandemic coronavirus disease 2019 (COVID-19) outbreak. Kurdistan Journal of Applied Research. 2020; 31-36.

[2] Balanzá-Martínez V, Atienza-Carbonell B, Kapczinski F, De Boni RB. Lifestyle behaviours during the COVID-19 time to connect. Acta Psychiatrica Scandinavica. 2020; 141(5): 399-400.

[3] Boness CL, Nelson M, Douaihy A. Motivational interviewing strategies for addressing COVID-19 vaccine hesitancy. 2021.

[4] Boreskie K, Hay J, Duhamel T. Preventing frailty progression during the COVID-19 pandemic. Journal of Frailty \& Aging. 2020; 1-2.

[5] Centers for Disease Control and Prevention. Coronavirus disease 2019 (COVID-19). 2020.

[6] Centers for Disease Control and Prevention. Coronavirus disease 2019 (COVID-19). Centers for Disease Control and Prevention. 2021.

[7] Chu D, Duda S, Solo K, Yaacoub S, Schunemann H. Physical distancing, face masks, and eye protection to prevent person-to-person transmission of SARS-Cov-2 and COVID-19: A systematic review and meta-analysis. Journal of Vascular Surgery. 2020; 72(4): 1500.

[8] DiMaio D, Enquist LW, Dermody TS. A new coronavirus emerges, this time causing a pandemic. Annual Review of Virology. 2020; 7(1): iii-v.

[9] Dinleyici EC, Borrow R, Safadi MA, Van Damme P, Munoz FM. Vaccines and routine immunization strategies during the COVID-19 pandemic. Human Vaccines \& Immunotherapeutics. 2020; 17(2): 400-407.

[10] Kirkcaldy RD, King BA, Brooks JT. COVID-19 and Postinfection immunity. JAMA. 2020; 323(22): 2245.

[11] Liu Y, Kuo R, Shih S. COVID-19: The first documented coronavirus pandemic in history. Biomedical Journal. 2020; 43(4): 328-333.

[12] Majumder M, Mandl KD. Early transmissibility assessment of a novel coronavirus in Wuhan, China. SSRN Electronic Journal. 2020.

[13] Olliaro P. What does 95\% COVID-19 vaccine efficacy really mean? The Lancet Infectious Diseases. 2021.

[14] Oronsky B, Gruber HE, Reiners W, Reid TR. A short discussion about the SARS-Cov-2 mrna-1273 vaccine. International Journal of Infectious Diseases. 2021; 104: 532-533. 
[15] Velavan, Thirumalaisamy P, Meyer Christian G. The COVID-19 epidemic. Trop Med Int Health. Mar 2020; 25(3): 278-280.

[16] World Health Organization. The race for a COVID-19 vaccine. WHO | World Health Organization. 2020.

[17] World Health Organization. Coronavirus update (Live): 13,953,344 cases and 592,778 deaths from CoVID-19 virus pandemic. Worldometer - real time world statistics. 2021.

[18] Wu YC, Ching-Sung C, Chen J, Chan Z, Yu-Jiun Y. The outbreak of COVID-19: An overview. Journal of the Chinese Medical Association. 2020; 83(2): 217-220. 